\title{
Guest Editorial Smart Systems and Architectures
}

\author{
Petar Šolić, Toni Perković, Ivan Marasović, Diego López-de-Ipiña, and Luigi Patrono
}

Smart systems and related supporting architectures both from hardware and software perspective are a vital part for future infrastructure deployment. To cope with further advancements, a series of integrations/compatibility issues still needs to be properly compensated while new advancements are presented. The aim of this Special issue is to gather different contributions that can be used in future smart systems architectures.

The paper "A Deep Learning Approach for Real-Time Analysis of Attendees' Engagement in Public Events" authored by Sujith Samuel Mathew, Manar AlKhatib, and May El Barachi presents a deep learning model enabling the accurate emotion analysis of crowded scenes containing complete and partially occluded faces, with different angles, aiming at improving smart city analytics. The proposed model was successfully tested using 3,750 images containing 22,563 faces, collected from a large consumer electronics trade show. It achieved an average accuracy of $90.6 \%$ when distinguishing between seven emotions (Happiness, smiling, laughter, neutral, sadness, anger, and surprise) in complete faces, and $86.16 \%$ accuracy in partially occluded faces.

The paper "Early Detection of External Neurological Symptoms through a Wearable Smart-Glasses Prototype", authored by Andrea Sciarrone, Igor Bisio, Chiara Garibotto, Fabio Lavagetto, Mehrnaz Hamedani, Valeria Prada, Angelo Schenone, Federico Boero, Gianluca Gambari, Marco Cereia, and Michele Jurilli contributes to the smart systems for medical sciences and healthcare. In particular, given the extreme diffusion of Alzheimer's disease (AD) and Parkinson's disease (PD), the demand for a solution to early detect neurological symptoms of such diseases strongly arose. This paper presents a prototype of wearable smart glasses able to detect the presence of Essential Tremor (ET) of the head and to count the number of Eye Blinks (EB) at the same time, in a transparent way with respect to the final user and used for purposes of eary detection of AD and PD. Numerical results demonstrate the reliability of the proposed approach: the proposed algorithms are able to i) correctly recognize the ET with an overall accuracy above $97 \%$ and ii) count the number of EBs with an overall error around $9 \%$.

Editorial approved on June 29, 2021 by Dinko Begušić. Date of publication: June 29, 2021

Petar Šolić, Toni Perković and Ivan Marasović are with the University of Split, Croatia. Diego López-de-Ipiña is with the University of Deusto, Bilbao, Spain. Luigi Patrono is with the University of Salento, Lecce, Italy. (e-mails: solic@fesb.hr, toperkov@fesb.hr, ivamaras@fesb.hr, dipina@deusto.es, luigi.patrono@unisalento.it).

Digital Object Identifier (DOI): 10.24138/jcomss-2021-0112
The paper "Fast-prototyping Approach to Design and Validate Architectures for Smart Home", authored by Teodoro Montanaro, Ilaria Sergi, Giovanni Marco Bisanti, Alessandro Cambò, and Luigi Patrono presents a solution that demonstrates how well-known fast-prototyping technologies like Node-RED, IBM Watson, Telegram, Raspberry Pi 4, and secured MQTT can contribute to develop complex systems facing the challenge of identifying platforms to fast prototype solutions satisfying new growing needs in IoT. The selected tools are used within a smart home context to support features inspired by people needs, and allow users to: a) consult real-time conditions (i.e., temperature, humidity, gas), b) remotely manage lights, c) save energy through a light management system based on user movements, d) remotely monitor the house through dedicated webcams, e) generate warning notifications in case of danger. Users can interact with the system through a web Node-RED dashboard and a Telegram bot. Differently from existing works, the feasibility of the implemented system and the efficacy of the exploited platforms are demonstrated through a running scenario extracted from a consolidated study on user needs in smart homes.

The paper "Demand Forecasting Tool For Inventory Control Smart Systems" authored by Fatima Zohra Benhamida, Ouahiba Kaddouri, Tahar Ouhrouche, Mohammed Benaichouche, Diego Casado-Mansilla, and Diego Lopez-deIpina presents an extensive literature review about demand forecasting methods for time-series data aiming at implementation of smart inventory management systems. Based on analysis results and findings, a new demand forecasting tool for inventory control is proposed. At first, a forecasting pipeline is designed to allow selecting the most accurate demand forecasting method. The validation of the proposed solution is executed on Stock\&Buy case study, a growing online retail platform. The evaluation process showed that the proposed tool achieves good forecasting accuracy by making the most appropriate choice while defining the forecasting method to apply for each product selection.

The paper "Observation of Enhanced Network Performance in IoT Process Control and Data Sensing with RINA", authlred by Bhushana Samyuel Neelam, and Benjamin A Shimray presents a prototype of IoT devices communicating on a new internet architecture i.e. recursive inter-networking architecture (RINA) which has eliminated hard-state handshaking procedures. The impact of RINA on the network performance in process control and data acquisition is observed in terms of latency variations, network jitter, and throughput. 
The results were compared with the network performance when the proposed prototype was communicating on TCP/IP. A Comparative analysis was provided to identify the improved network performance in RINA.

The paper "Optimized method for locating the source of voltage sags" authored by Jose Lima Filho, Fabbio Anderson da Silva Borges, Ricardo de Andrade Lira Rabelo, Ivan Saraiva Silva and Antonio Oseas de Carvalho Filho, is dealing with monitoring in smart grids. This monitoring generates a large volume of data (Big data) and as a result, multiple challenges arise due to the properties of this data such as volume, variety, and velocity. This work presents an optimization through genetic algorithm to select meters which already exist in the smart grid, using a voltage sag location method to reduce the data obtained and analyzed throughout the localization process. Optimization was evaluated through a comparison with a nonoptimized localization method, this comparison showed a difference between the hit rates of less than $1 \%$.

\section{ACKNOWLEDGEMENT}

This work has been supported in part by Croatian Science Foundation under the project "Internet of Things: Research and Applications", UIP-2017-05-4206, Croatia.

\section{GUEST EDITORS}

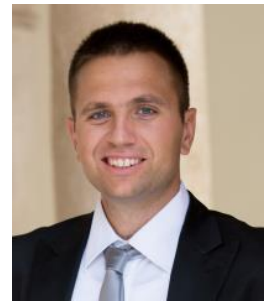

Petar Šolić (psolic@fesb.hr) received his M.S. and $\mathrm{Ph} . \mathrm{D}$. degrees, both in computer science, from the University of Split in 2008 and 2014, respectively. He is currently employed at the Faculty of Electrical Engineering, Mechanical Engineering and Naval Architecture (FESB), University of Split, Croatia, as an associate professor in the Department of Communication and Information Technologies. His research interests include information technologies, and RFID technology and its application. In 2016 and 2020 he was awarded with National prize for science.

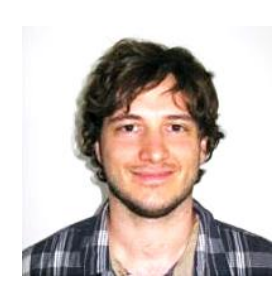

Toni Perković (toperkov@fesb.hr) received the Dipl. Ing. degree in telecommunications and electrical engineering and the Ph.D. degree in computer science from the University of Split, Split, Croatia, in 2007 and 2013, respectively. He is currently employed as an Associate Professor with the Faculty of Electrical Engineering, Mechanical Engineering and Naval Architecture, University of Split. His research interests include the usability, design, and analysis of security protocols for wireless networks, and the usability and design of secure authentication protocols.

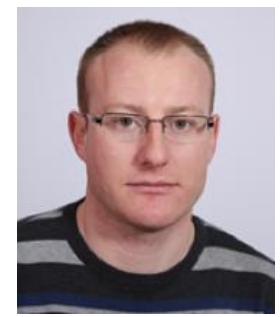

Ivan Marasović (ivamaras@fesb.hr) is currently employed as Associate Professor at the Faculty of Electrical Engineering, Mechanical Engineering and Naval Architecture (FESB), University of Split, Croatia, Department of Electronics and Computing, Group for nanoelectronics and photovoltaic conversion. He received the Dipl.
Ing. degree in automation and electrical engineering from the University of Split, Croatia, in 2007, and the PhD degree in Electronics (nanostructures) from the University of Split, Croatia, in 2011. His research interests include the solar cells and photovoltaic conversion, nanoeletronics, photovoltaic systems, embedded systems - Internet of Things and industry automation systems.

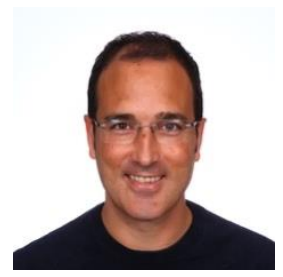

Diego López-de-Ipiña (dipina@deusto.es) received the Ph.D. degree from the University of Cambridge, U.K., in 2002. His Ph.D. dissertation entitled visual sensing and middleware support for sentient computing. $\mathrm{He}$ is currently an Associate Professor and a Principal Researcher of the MORElab/DEUSTEK Group (http://morelab.deusto.es/). He has participated in several big consortium-based research european (PARITY, BD4QoL, EDI, SIMPATICO, CITY4AGE, GREENSOUL, WELIVE, MOVESMART, IES CITIES, MUGGES, SONOPA, CBDP, GO$\mathrm{LAB}$, and LifeWear) involving the adoption of semantic web, social data mining, linked open data, smart cities, open government, and NGI to novel AmI-related application areas such as urban computing, sustainable computing or AAL. He is the Project Coordinator for the European Data Incubator (EDI) (http://edincubator.eu) H2020 Project. $\mathrm{He}$ has more than 200 publications in relevant international conferences and journals on Ubiquitous Computing, Semantic Web, Middleware, Smart Cities, and AmI, including more than 65 JCRindexed journal articles. His main research interests are pervasive computing, the Internet of Things, semantic service middleware, open linked data, social data mining, and mobile-mediated and tangible humanenvironment interaction.

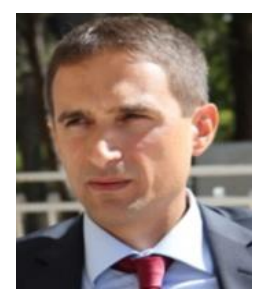

Luigi Patrono (luigi.patrono@unisalento.it) received his MS in Computer Engineering from University of Lecce, Lecce, Italy, in 1999 and $\mathrm{PhD}$ in Innovative Materials and Technologies for Satellite Networks from ISUFI-University of Lecce, Lecce, Italy, in 2003. He is an Assistant Professor of Computer Network at the University of Salento, Lecce, Italy. His research interests include RFID, EPCglobal, Internet of Things, Wireless Sensor Networks, and design and performance evaluation of protocols. He is Organizer Chair of the international Symposium on RFID Technologies and Internet of Things within the IEEE SoftCOM conference. He is Guest Editor of the Special Issue on RFID Technologies \& Internet of Things. He is author of more than 120 scientific papers published on international journals and conferences and four chapters of books with international diffusion. 